\title{
ANÁLISIS BIBLIOMÉTRICO DE LA PRODUCCIÓN CIENTÍFICA BIOMÉDICA EN LA REGIÓN DE ICA, PERÚ. 1998-2010*
}

\author{
C. Hugo Arroyo-Hernández ${ }^{1,3, a}$, Beatriz Zukerán-Medina ${ }^{1, a}$, Ubaldo E. Miranda-Soberón ${ }^{1,2, b}$
}

1. Facultad de Medicina, Universidad Nacional San Luís Gonzaga. Ica, Perú

2. Hospital Regional de Ica, Ministerio de Salud. Ica, Perú.

3. Centro de Salud de Luricocha, Dirección Regional de Salud de Ayacucho. Huanta, Perú.

a. Médico Cirujano; b. Médico Pediatra

\section{RESUMEN}

Objetivo: Determinar mediante un análisis bibliométrico la producción científica biomédica en la región de Ica, Perú entre los años 1998-2010. Material y métodos: Estudio de tipo bibliométrico, observacional, transversal y descriptivo, se realizo una búsqueda en internet de artículos publicados en revistas científicas biomédicas indizadas a un base de datos, usando la estrategia de búsqueda por afiliación y palabras clave relacionadas con instituciones en ciencias de la salud de la región Ica, la información obtenida fue procesada y expresada en frecuencias. Resultados: Se encontraron 56 publicaciones de las cuales el 80,3\% fueron en revistas nacionales, del total de publicaciones el 66\% fueron artículos originales, el área de mayor interés en investigación fue la salud pública con el $44,6 \%$, según el diseño de investigación usado el $77,5 \%$ fueron de tipo transversal y descriptivo, la institución con mayor producción científica fue la Facultad de Ciencias de la Universidad Nacional San Luis Gonzaga, el $41,5 \%$ de autores estaban afiliados a otra región; del total de autores afiliados a una institución de la región Ica el 67,6\% eran hombres, la profesión más frecuente fue médicos y biólogos con $56,4 \%$ y 29,3\% respectivamente. Conclusiones: Es necesario que las instituciones en ciencias de la salud de la región Ica, consideren la importancia de la publicación como indicador de producción científica, capacitando y fomentando una cultura de publicación en sus miembros. (Rev. méd. panacea $2011 ; 1: 2-8)$.

Palabras clave: Indicadores de producción científica, Ciencias de la salud, Investigación biomédica, Perú (fuente: DeCS BIREME).

\section{BIBLIOMETRIC ANALYSIS OF BIOMEDICAL SCIENTIFIC PRODUCTION IN THE REGION OF ICA, PERU. 1998-2010}

\begin{abstract}
Objective: To determine, through a bibliometric analysis biomedical scientific production in the region of Ica, in the years 1998-2010. Material and methods: Bibliometric study, observational, cross sectional, we made a search online database of articles published by institutions in the health sciences in the Ica region using the search strategy for affiliation and the keywords, information obtained was processed and expressed in frequencies. Results: We found 56 publications of which $80.3 \%$ were in national magazines, publications of the total $66 \%$ were original articles, the largest area of research interest in public health was $44.6 \%$, according to the design research used the $77.5 \%$ were cross-sectional and descriptive, the institution with scientific production was the Facultad de Ciencias of the Universidad Nacional San Luis Gonzaga, $41.5 \%$ of authors were affiliated with another region of the total authors affiliated with an institution of the Ica region $67.6 \%$ were men, the medical profession was the most frequent and biologists with $56.4 \%$ and $29.3 \%$ respectively. Conclusions: It is necessary that institutions in health sciences in the lca region, consider the importance of the publication as an indicator of scientific production, fostering a culture of publication to its members.(Rev. méd. panacea $2011 ; 1: 2-8)$.
\end{abstract}

Key words: Scientific publication indicators, Health sciences, Biomedical research, Peru (source: MeSH NLM).

* Los resultados preliminares de la investigación se publicaron en: Arroyo-Hernández CH, Zukerán-Medina EB, Miranda-Soberón UE. Características de la producción científica biomédica en Ica, Perú 1998-2007. Rev Peru Med Exp Salud Pública 2009; 26(2):203-6.

\section{INTRODUCCIÓN}

Las publicaciones en revistas científicas constituyen el más importante indicador de producción científica, permitiendo el intercambio de conocimientos mediante su difusión continua, generando además la evidencia necesaria para la toma de decisiones que permitan solucionar problemas de salud específicos ${ }^{(1,2)}$.

Los estudios bibliométricos aplican métodos matemáticos y estadísticos a toda literatura de carácter científico, en la 
actualidad las bases de datos disponibles en internet son la principal herramienta utilizada para medir la producción científica $^{(3,4)}$.

La escasa contribución del Perú a la producción científica Latinoamericana, observada en estudios bibliométricos, es un problema asociado a la pobre inversión en investigación científica y tecnológica de países en vías de desarrollo como el nuestro ${ }^{(5,6)}$.

Siendo además la investigación científica, una función obligatoria que debe ser asumida por sus docentes y alumnos, según la legislación universitaria peruana, lo poco inclinados a escribir y compartir experiencias científicas, clínico o académicas es resultado de la poca exigencia de las universidades peruanas por la publicación ${ }^{(7)}$.

En la región de Ica, una pobre formación en investigación recibida en pre grado y la ausencia de sólidas líneas de investigación dan como resultado el escaso número de investigaciones realizadas aun en el post grado, esto sumado a la falta de capacitación en temas de redacción científica y la migración de los profesionales que sirvan como modelos formativos en publicación, se convierte en un problema que debe ser afrontado por las autoridades universitarias $^{(8)}$.

El objetivo de esta investigación es determinar mediante un análisis bibliométrico las características de la producción científica biomédica en la región de lca, durante los años 1998 y 2010.

\section{MATERIAL Y MÉTODOS}

Se realizó un estudio de tipo bibliométrico, observacional, transversal y descriptivo.

Se elaboró una estrategia de búsqueda en internet que incluyera artículos de investigación, reporte de casos y cartas al editor publicados en una revista científica biomédica indizada a una base de datos durante los años 1998 y 2010; los artículos publicados debían contar con la participación de al menos un autor afiliado a una institución en ciencias de la salud de la región lca.

Se consideró como artículos originales a las investigaciones realizadas usando el método científico y presentadas según el formato acordado por el ICMJE, que consta de Introducción, Materiales y métodos, Resultados Y Discusión (IMRYD); como comunicaciones cortas u originales breves a las investigaciones que usan el método científico, pero que la revista considera que no deben figurar como artículos originales, pues representan investigaciones preliminares o cuya metodología no permite sacar conclusiones definitivas; como Reporte de casos a los informes de casos aislados o series de casos, que describen la presentación clínica ${ }^{(9)}$.

Las publicaciones debían encontrase disponibles en resumen o texto completo y estar relacionadas con las ciencias de la salud pudiendo ser de acuerdo al nivel y objeto de análisis a) Biomédico, sí se ocupa de las condiciones y los procesos de la salud y la enfermedad a nivel individual y subindividual (celular, molecular, etc.), b) Clínico, cuando suele enfocarse hacia el estudio de las respuestas terapéuticas, preventivas o para rehabilitación que se aplican al individuo y c) Salud Pública, cuando se ocupa del estudio de las necesidades de salud y las repuestas sociales a dichas necesidades a nivel poblacional; o el área de d) educación médica.

Consideramos como base de datos aquellos índices que incluyen revistas científicas periódicas evaluadas a través de un proceso continuo (arbitraje por pares, consejo editorial idóneo, actualización de sus presentaciones, etc). Para verificar y corroborar la indezación de cada revista se accedió a la página web de cada institución editora o de la propia revista.

Como primer paso en la recolección de las publicaciones se uso el motor de búsqueda de Google Scholar (www.scholar.google.com) ingresando palabras clave relacionadas con instituciones en ciencias de la salud de la región de Ica.

Similar estrategia se utilizo posteriormente en los motores de búsqueda de las base de datos de SciELO, Scientific Electronic Library Online, (www.scielo.org); LILACS, Literatura Latinoamericana y del Caribe en Ciencias de la Salud (www.bireme.br), LIPECS, Literatura Peruana en Ciencias de la Salud. (http://www.upch.edu.pe/vrinve/dugic/ index.asp) y Medline (US National Library of Medicine) cuyo motor de búsqueda es Pubmed (www.ncbi.nlm.nih.gov).

En caso de no acceder al texto completo de un artículo publicado en una revista científica nacional indizada a una de estas base de datos se ingreso al repositorio electrónico del Sistema de Biblioteca de la Universidad Nacional Mayor de San Marcos, SISBIB-UNMSM (www.sisbib.unmsm.edu.pe/bibvirtual), realizando una búsqueda del articulo mediante el nombre de la revista, año de publicación, volumen y numero.

Las variables objeto de análisis, que incluían las características del artículo publicado, los autores y la revista se ingresaron en una hoja de cálculo diseñada en el software Microsoft Excel 2009, para luego ser procesada y expresada en frecuencias y porcentajes. 


\section{RESULTADOS}

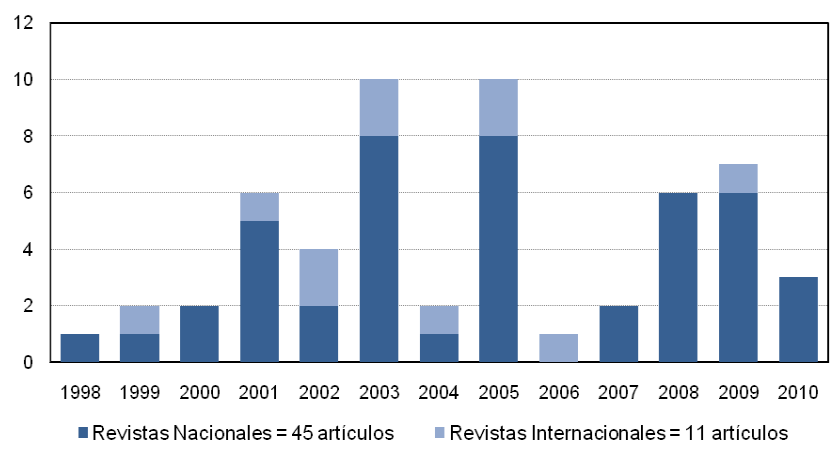

Figura 1. Producción científica biomédica en la región de Ica, Perú 1998-2010.

Se encontró un total de 56 publicaciones científicas en trece años distribuidos de forma heterogénea, siendo los años 2003 y 2005 con el mayor número de publicaciones y 1998 y 2006 con la mínima, el $80,4 \%$ del total de artículos publicados se realizaron en revistas nacionales (Figura 1).

De los 24 títulos de revistas científicas biomédicas halladas $15(62,5 \%)$ correspondían a revistas nacionales, siendo la Revista Peruana de Medicina Experimental y Salud Pública (Rev Peru Med Exp Salud Pública) donde se encontró el mayor número de artículos publicados (25\%), seguido por la Revista Ciencia e Investigación Medico Estudiantil Latinoamericana (CIMEL). El $53,3 \%$ de las revistas nacionales se encontraban desactualizadas, o fuera de circulación, del total de artículos publicados en revistas nacionales el 78,6\% (44) están disponibles en la colección digital del SISBIB-UNMSM, 64,3\% (36/56) en la base de datos de LIPECS y $50 \%$ (28/56) en LILACS, todas las revistas nacionales indezadas a SciELO estaban actualizadas, las únicas revistas nacionales indizadas a una base de datos de visibilidad internacional como Medline fueron la Revista de Gastroenterología del Perú (Rev Gastroenterol Peru) y desde el año 2009 la Rev Peru Med Exp Salud Pública; de las once revistas científicas internacionales el mayor número de artículos se encontraron en la revista Parasitología Latinoamericana (Parasitol Latinoam), anteriormente llamada Boletín Chileno de parasitología y Parasitología al día, contando todas con presencia en Medline (Tabla 1).

Las características de los 56 artículos publicados fueron, según su tipo: Artículos originales $(66,1 \%)$, Reporte de casos y Carta al editor, ambas con $14,3 \%$, y Comunicación corta con 5,4\%; las áreas de mayor interés en investigación fueron la Salud pública y la Biomédica con $44,6 \%$ y $23,2 \%$ respectivamente; según el diseño de investigación usado

Tabla 1. Características de los artículos publicados en revistas científicas biomédicas indizadas, 1998-2010.

\begin{tabular}{|c|c|c|c|c|c|}
\hline Titulo abreviado de la revista & Artículos & (\%) & Periodicidad & Actualizada & Indización \\
\hline \multicolumn{6}{|l|}{ Revistas Nacionales } \\
\hline Rev Peru Med Exp Salud Pública* & 14 & $(25,0)$ & Trimestral & $\mathrm{Si}$ & Medline/SciELO-Perú/LILACS/LIPECS/SISBIB-UNMSM \\
\hline CIMEL & 5 & $(8,9)$ & Semestral & $\mathrm{Si}$ & SISBIB-UMMSM \\
\hline Enf Torax & 4 & $(7,1)$ & Cuatrimestral & No & LILACS/LIPECS/SISBIB-UMMSM \\
\hline Rev Peru Parasitol & 4 & $(7,1)$ & Semestral & No & SISBIB-UMMSM \\
\hline Rev Gastroenterol Peru & 3 & $(5,4)$ & Trimestral & $\mathrm{Si}$ & Medline/SciELO-Perú/LILACS/LIPECS/SISBIB-UNMSM \\
\hline Rev Peru Enf Infec Trop & 3 & $(5,4)$ & Semestral & No & LIPECS/SISBIB-UMMSM \\
\hline An Fac Med & 2 & $(3,6)$ & Trimestral & $\mathrm{Si}$ & SciELO-Perú/LILACS/LIPECS/SISBIB-UNMSM \\
\hline Acta Med Peru & 2 & $(3,6)$ & Cuatrimestral & $\mathrm{Si}$ & SciELO-Perú/LILACS/LIPECS/SISBIB-UNMSM \\
\hline Rev Soc Peru Med Interna & 2 & $(3,6)$ & Trimestral & No & LILACS/LIPECS/SISBIB-UMMSM \\
\hline Ginecol \& Obstet & 1 & $(1,8)$ & Trimestral & $\mathrm{Si}$ & LIPECS/SISBIB-UMMSM \\
\hline Rev Peru Biol & 1 & $(1,8)$ & Semestral & $\mathrm{Si}$ & SciELO-Perú/LILACS/LIPECS/SISBIB-UNMSM \\
\hline Rev Peru Cardiol & 1 & $(1,8)$ & Trimestral & No & LIPECS/SISBIB-UMMSM \\
\hline Gac odontol & 1 & $(1,8)$ & Semestral & No & LIPECS \\
\hline Acta Cancerol & 1 & $(1,8)$ & Semestral & No & LIPECS/SISBIB-UMMSM \\
\hline Rev Acad Peru salud & 1 & $(1,8)$ & Semestral & No & LIPECS/SISBIB-UMMSM \\
\hline \multicolumn{6}{|l|}{ Revistas Internacionales } \\
\hline Parasitol Latinoam ** & 5 & $(45,5)$ & Semestral & No & Medline/SciELO \\
\hline Research in Microbiology & 1 & $(9,1)$ & Mensual & $\mathrm{Si}$ & ISI/Medline \\
\hline Am J Trop Med Hyg & 1 & $(9,1)$ & Mensual & $\mathrm{Si}$ & ISI/Medline \\
\hline Plos ONE & 1 & $(9,1)$ & No declarada & $\mathrm{Si}$ & ISI/Medline \\
\hline Vaccine & 1 & $(9,1)$ & Semanal & $\mathrm{Si}$ & ISI/Medline \\
\hline Rev Panam Salud Publica & 1 & $(9,1)$ & Bimestral & $\mathrm{Si}$ & Medline/SciELO/LILACS \\
\hline Cad Saude Pública & 1 & $(9,1)$ & Mensual & $\mathrm{Si}$ & ISI/Medline/SciELO \\
\hline
\end{tabular}

* Indizada a Medline desde el año 2009, ${ }^{* *}$ Títulos anteriores Boletín Chileno de parasitología y Parasitología al día. 
Tabla 2. Características de los artículos publicados.

\begin{tabular}{lcc}
\hline \multicolumn{1}{c}{ Características } & $\mathbf{N}=\mathbf{5 6}$ & $(\%)$ \\
\hline Tipo de articulo & 37 & $(66,1)$ \\
$\quad$ Artículo original & 8 & $(14,3)$ \\
Reporte de casos & 8 & $(14,3)$ \\
$\quad$ Carta al editor & 3 & $(5,4)$ \\
$\quad$ Comunicación corta & & \\
Área de investigación & 25 & $(44,6)$ \\
$\quad$ Salud pública & 13 & $(23,2)$ \\
$\quad$ Biomédica & 11 & $(19,6)$ \\
$\quad$ Clínica & 7 & $(12,5)$ \\
$\quad$ Educación Médica & & \\
Diseño de investigación (n=40) & & \\
$\quad$ Transversal descriptivo & 31 & $(77,5)$ \\
$\quad$ Transversal analítico & 3 & $(7,5)$ \\
Casos y controles & 3 & $(7,5)$ \\
Experimental & 3 & $(7,5)$ \\
\hline
\end{tabular}

en los artículos originales y comunicación corta el 77,5\% correspondieron a diseños de tipo transversal descriptivo (Tabla 2).

De acuerdo a las instituciones con mayor producción científica en la región Ica, la Universidad Nacional San Luis Gonzaga tuvo una participación en el total de artículos publicados del 66,1\% (37/56), siendo su Facultad de Ciencias la de mayor contribución (35,7\%), seguida por la Facultad de Medicina $(23,2)$, y la menor la Facultad de Enfermería con solo un artículo, en diez artículos no se encontró la facultad a la que pertenecían los autores; el segundo grupo de instituciones con mayor producción científica fueron los Hospitales (15/56) en su mayoría autores afiliados al Hospital Regional de Ica. El porcentaje de colaboración con instituciones de otras regiones fue del 67,9\% (38/56) encontrándose mayor participación de instituciones de la ciudad de Lima $(46,4 \%)$ como el Instituto Nacional de Salud y la Dirección General de Epidemiología, la colaboración con instituciones de otras provincias fue similar con las de instituciones extranjeras (Tabla 3).

Se contabilizaron un total de 253 autores, de acuerdo al número de autores por artículo el $37,5 \%$ estuvo conformado por más de cinco, el mínimo de autores fue uno y el máximo diez, el $58,5 \%$ de los autores indico su filiación con una institución de la región Ica y el $41,5 \%$ a una institución de otra región; según el sexo de los autores con filiación institucional en la región Ica el $67,6 \%$ son hombres, la profesión con mayor participación fueron los médicos y biólogos ambos con $56,4 \%$ y $29,3 \%$ respectivamente, se encontró un $17,8 \%$ de participación estudiantil en el total de artículos publicados (Tabla 4).
Tabla 3. Instituciones con mayor producción científica, 1998-2010.

\begin{tabular}{|c|c|c|}
\hline Nombre & $N=56$ & (\%) \\
\hline \multicolumn{3}{|l|}{ Instituciones en Ica } \\
\hline \multicolumn{3}{|l|}{ Universidad Nacional San Luis Gonzaga $(n=37)$} \\
\hline Facultad de Ciencias & 20 & $(35,7)$ \\
\hline Facultad de Medicina & 13 & $(23,2)$ \\
\hline Facultad de Veterinaria & 3 & $(5,4)$ \\
\hline Facultad de Enfermería & 1 & $(1,8)$ \\
\hline No se indica facultad & 10 & $(17,9)$ \\
\hline \multicolumn{3}{|l|}{ Hospitales $(n=15)$} \\
\hline Hospital Regional de Ica & 10 & $(17,9)$ \\
\hline Hospital EsSalud & 5 & $(8,9)$ \\
\hline Hospital Santa María del Socorro & 2 & $(3,6)$ \\
\hline Dirección Regional de Salud de Ica & 6 & $(10,7)$ \\
\hline \multicolumn{3}{|l|}{ Instituciones en Lima $(n=26)$} \\
\hline Instituto Nacional de Salud & 9 & $(16,1)$ \\
\hline Dirección General de Epidemiologia & 8 & $(14,3)$ \\
\hline Universidad Nacional Mayor de San Marcos & 7 & $(12,5)$ \\
\hline Universidad Peruana Cayetano Heredia & 3 & $(5,4)$ \\
\hline Hospitales & 3 & $(5,4)$ \\
\hline Organismos No Gubernamentales & 2 & $(3,6)$ \\
\hline Otras & 2 & $(3,6)$ \\
\hline \multicolumn{3}{|l|}{ Instituciones en otras provincias $(n=6)$} \\
\hline Universidades & 4 & $(7,1)$ \\
\hline Dirección Regional de Salud de Ayacucho & 2 & $(3,6)$ \\
\hline Dirección Regional de Salud de Huancavelica & 2 & $(3,6)$ \\
\hline \multicolumn{3}{|l|}{ Instituciones Extranjeras $(n=6)$} \\
\hline Universidades en Latinoamérica & 5 & $(8,9)$ \\
\hline Universidades en Norteamérica & 4 & $(7,1)$ \\
\hline Organismos No Gubernamentales & 1 & $(1,8)$ \\
\hline
\end{tabular}

En la Tabla 4 se muestran los resultados de los autores afiliados a una institución de la región lca con mayor número de artículos publicados en revistas científicas indizadas y quienes contribuyeron con más del $50 \%$ del total de la producción científica biomédica en la región, el autor de mayor producción estaba afiliado a la Facultad de Ciencias y tres a la Facultad de Medicina de la Universidad Nacional San Luis Gonzaga uno de los cuales era estudiante, dos de los autores fueron afiliados posteriormente a una institución en la ciudad de Lima

\section{DISCUSIÓN}

Los resultados encontrados en esta investigación permiten evidenciar la verdadera producción científica realizada por las instituciones en ciencias de la salud de la región de lca. 
Tabla 4. Características de los autores.

\begin{tabular}{lcc}
\hline \multicolumn{1}{c}{ Características } & N & $(\%)$ \\
\hline Autores por artículo* $(\mathbf{n}=56)$ & 7 & $(12,5)$ \\
Uno & 7 & $(12,5)$ \\
Dos & 12 & $(21,4)$ \\
Tres & 9 & $(16,1)$ \\
Cuatro & 21 & $(37,5)$ \\
Más de cinco & & \\
Filiación institucional ( $\mathbf{n}=\mathbf{2 5 3})$ & 148 & $(58,5)$ \\
En la región de Ica & 105 & $(41,5)$ \\
Otra región & & \\
Sexo (n=148) & 100 & $(67,6)$ \\
Hombre & 48 & $(32,4)$ \\
Mujer & & \\
Profesión autores de la región Ica ( $\mathbf{n}=\mathbf{1 4 8})$ & 75 & $(56,4)$ \\
Médico & 39 & $(29,3)$ \\
Biólogo & 5 & $(3,8)$ \\
Odontólogo & 5 & $(3,8)$ \\
Veterinario & 2 & $(1,5)$ \\
Enfermera & 7 & $(5,3)$ \\
No se indica & 10 & $(17,8)$ \\
Artículos publicados con participación & & \\
estudiantil ( $\mathbf{n}=\mathbf{5 6})$ & & \\
\hline
\end{tabular}

* promedio de autores por artículo 4,5; Min 1 - Max 10

La producción científica en los trece años revisados no es homogénea, presentando marcadas variaciones en su distribución no asegurando una tendencia creciente en los próximos años, por lo que consideramos necesario comenzar a precisar metas cuantificables que permitan trazar objetivos para mejorar los indicadores de producción científica, siendo esto responsabilidad de las instituciones locales que ejercen la gerencia de la ciencia.

El uso de la bibliometría y de sus herramientas constituye una valiosa metodología de evaluación científica, aunque se ha de tener cuidado al comparar el número de publicaciones, en especial las realizadas en nuestra región, debido a que sobre este influyen diversos factores socioeconómicos y culturales ${ }^{(10)}$, nuestros resultados permiten demostrar que la producción científica biomédica de la región es fácilmente superado por publicaciones realizadas por estudiantes de medicina de Chile en revistas médicas indezadas a SciELO, que llegan de 50 a 70 por año ${ }^{(11)}$, y representa en promedio solo el $8 \%$ de la producción científica realizada por docentes de la Facultad de Medicina de la Universidad Nacional Mayor de San Marcos durante diez años ${ }^{(12)}$.

Gran parte de las publicaciones realizada por autores afiliados a una institución de salud de la región Ica se encontraron en revistas biomédicas indezadas en base de datos nacionales y en menor medida las internacionales como LILACS o Medline, es importante mencionar además la labor del Sistema de Bibliotecas de la Universidad Nacional Mayor de San Marcos y su colección digital de revistas que permitió encontrar y acceder a textos completos en revistas nacionales desactualizadas 0 aquellas donde SciELO-Perú o Medline no incluyen dentro de sus años de indización. Sin embargo el alto porcentaje de publicaciones en revistas desactualizadas es una muestra del desconocimiento de los autores locales sobre los criterios para escoger una adecuada revista donde publicar, siendo también responsabilidad de las asociaciones e instituciones que dirigen estas revistas mantener la periodicidad de sus ediciones y estándares de calidad $^{(14,15)}$.

Aunque no fue objeto del estudio determinar la calidad de los artículos publicados, una manera de medir su impacto es mediante el número de citas recibidas, es de decir las veces que un artículo es mencionado en las referencias bibliográficas de otras publicaciones, es por ello que cuando un investigador profesional decide publicar su estudio, espera hacerlo en una revista que le permita llegar a una mayor cantidad de público que pueda usar su investigación, siendo de conocimiento que los artículos publicados en revistas internacionales indizadas a Medline e ISI (Institute for Scientific Information) así como investigaciones en el área biomédica o de las ciencias básicas alcanzan un mayor número de citas, aportando a su vez mayor prestigio a sus autores, algunos ejemplos de revistas de alto impacto donde se encontró artículos publicados por autores de la región son Research in Microbiology, American Journal of Tropical Medicine and Hygiene, Plos One o Vaccine; aunque estas contribuciones pueden ser consideradas ocasionales debido a su baja frecuencia, su presencia puede deberse más bien a la colaboración de autores locales con los intereses de investigadores extranjeros con una trayectoria más amplia de publicaciones, sin embargo la experiencia alcanzada por los autores locales es importante ${ }^{(13)}$.

Siendo los artículos originales el tipo de publicación más frecuente muchas de las investigaciones que se realizan como tesis en facultades de ciencias de la salud y escuelas de postgrado de la universidad no terminan con su publicación en una revista científica indizada, lo que demostraría una falta de capacitación y valoración del trabajo realizado, demandando una intervención y seguimiento por parte de los responsables de estas instituciones. Asimismo los bajos porcentajes de reporte de casos y cartas al editor reflejan el desconocimiento de oportunidades para compartir experiencias científicas y del quehacer de los profesionales en ciencias de la salud ${ }^{(9)}$ 
Tabla 5. Autores con mayor número de publicaciones afiliados a una institución en ciencias de la salud de la región de lca, Perú.

\begin{tabular}{lccl}
\hline \multicolumn{1}{c}{ Nombre } & N = 56 & $(\%)$ & \\
\hline Rufino Cabrera* & 12 & $(21,4)$ & Biólogo, Facultad de Ciencias, Universidad Nacional San Luis Gonzaga \\
Hugo Arroyo-Hernández & 9 & $(16,1)$ & Estudiante de Medicina, SOCEMI**, Facultad de Medicina, Universidad Nacional San Luis Gonzaga \\
Luis Suarez-Ognio* & 6 & $(10,7)$ & Médico, Dirección Regional de Salud, Ministerio de Salud. Ica, Perú \\
Julio Torres-Chang & 5 & $(8,9)$ & Médico, Hospital Regional de Ica, Facultad de Medicina, Universidad Nacional San Luis Gonzaga \\
Ubaldo Miranda-Soberón & 5 & $(8,9)$ & Médico, Hospital Regional de Ica, Facultad de Medicina, Universidad Nacional San Luis Gonzaga \\
\hline
\end{tabular}

* Actualmente afiliados a la Dirección General de Epidemiología de Lima, MINSA; ** Sociedad Científica de Estudiantes de Medicina de Ica.

Según el diseño de investigación el mayor número artículos fueron de tipo transversal descriptivo y en menor porcentaje de casos control o experimentales, esta es una característica común a la hallada en una investigación bibliométrica realizada por Romaní y colaboradores titulada uso de de los métodos estadísticos en artículos originales de cinco revista biomédicas peruanas durante el periodo 2002-2009, en el mismo estudio se reconoce además que el uso de diseños de tipo transversal descriptivo y analítico son usados con mayor frecuencia en publicaciones del área de la salud pública ${ }^{(16)}$.

Con respecto a la escasa contribución de estudios de tipo experimental en una comunicación previa consideramos fundamental para el desarrollo de nuestras universidades la implementación de la investigación en ciencias básicas desde el pregrado sin embargo la escasa o falta absoluta de equipamiento y personal tecnificado para su uso, limitaría el nivel y calidad de las investigaciones que se pudiera realizar ${ }^{(17)}$.

Aunque podríamos manifestar que son pocos los autores de la región con conocimientos para la elaboración de estudios que permitan la contrastación de hipótesis mediante el uso de estadística analítica, debemos mencionar además que no es necesario el uso de diseños estadísticos muy elaborados para que una investigación pueda culminar en una publicación, siempre y cuando la metodología guarde coherencia estadística con los resultados que se quiera mostrar, estando además bien redactado y se respeten las normas éticas en publicación.

En la actualidad la investigación científica, no se realiza de manera aislada sino que requiere la participación de diversos investigadores que conformen redes de colaboración entre instituciones, regiones o países; en nuestro estudio encontramos mayor colaboración de instituciones del Ministerio de Salud en la ciudad de Lima y en menor número instituciones de provincias y del extranjero, esta colaboración con autores de otras regiones es una ventaja que permite superar limitaciones de recursos materiales, técnicos o profesionales, por ello se sugiere promover una mayor colaboración institucional con otras regiones del país y del extranjero ${ }^{(13)}$.

Las características en el promedio de autores por artículo de investigación, predominio en el sexo de los hombres y de la profesión médica guardan relación con un estudio similar realizado en una provincia de Cuba ${ }^{(21)}$.

Nuestro estudio presenta una evidencia importante de participación estudiantil en la publicación de artículos científicos, la misma que se viene reportando en otras universidades, como lo muestra Huamaní y colaboradores quienes encontraron un 4,5\% de participación estudiantil en revistas médicas peruanas indizadas a SciELO-Perú durante los años 1997 y $2005^{(18)}$. Al respecto en el Perú así como en otros países de Latinoamérica las sociedades científicas estudiantiles están desempeñando un rol fundamental, al comenzar a tomar políticas que consideran a las publicaciones en revistas indezadas como su principal indicador de producción científica, capacitando a sus miembros mediante cursos de redacción científica y proceso de publicación ${ }^{(19,20)}$.

Si bien la producción científica biomédica en nuestra región es escasa, la universidad debe propiciar la difusión y visibilidad de estas contribuciones, permitiendo su crítica, reconocimiento y motive a miembros de la comunidad académica de nuestra región; una forma directa y sencilla es la incorporación de espacios en la página web de cada institución, que permita el acceso a través de hipervínculos a los artículos publicados por su institución, un ejemplo de esto es la pagina web de la Facultad de Medicina de la Universidad José Faustino Sánchez Carrión de Huacho (www.med-unjfsc.edu.pe/publicaciones/), en nuestra región el único portal en internet que viene realizando esto es la Sociedad Científica de Estudiantes de Medicina de Ica (www.socemi.blogstpot.com).

La falta de una cultura de publicación científica no es exclusiva en profesionales de ciencias de la salud de nuestra región, sin embargo, el interés y habito hacia la publicación se adquiere en base a experiencias previas, siendo fundamental que la enseñanza universitaria fomente 
no solo la investigación, sino que también incluya desde el pregrado la capacitación en temas de redacción científica y proceso de publicación, estudios similares en otras regiones del Perú con similares características podrían contribuir a mostrar sus indicadores de producción científica.

Recibido: 08-4-11 Aprobado: 29-4-11

Financiamiento: El estudio fue autofinanciado

Conflictos de interés: La versión final de esta investigación es publicada en esta revista con la finalidad de compartir los resultados a nivel local.

\section{REFERENCIAS BIBLIOGRÁFICAS}

1. De Meis $L$, De Cassia $R$, Machado $P$, Fonseca $L$, Lustosa P, Caldeira MT. Cienciometría y evaluaciones por los propios investigadores. Interciencia. 1992;(17): 40-43.

2. Bordons $\mathbf{M}$, Zulueta MA. Evaluación de la actividad científica a través de indicadores bibliométricos. Rev Esp Cardiol. 1999;52(10):790-800.

3. Moya-Anegón F, Herrero-Solana V, Vargas-Quesada B, Chinchilla-Rodríguez Z, Corera-Álvarez E, Muñoz-Fernández $\mathbf{F}$, et al. Atlas de la ciencia Española: Propuesta de un sistema de información científica. Rev Esp Doc Cient. 2004; 27(1):11-29.

4. Sotolongo-Aguilar G, Guzmán-Sánchez MV, Carrillo H. ViBLIOSOM: visualización de información bibliométrica mediante el mapeo autoorganizado. Rev Esp Doc Cient. 2002; 25(4):477-84.

5. De Moya-Anegón F, Herrero-Solana V. Visibilidad internacional de la producción científica iberoamericana en biblioteconomía y documentación (1991-2000). Ci Inf Brasilia. 2002; 31(3):54-65.

6. Sancho R, Morillo F, De Filippo D, Gómez I, Fernández MT. Indicadores de colaboración científica inter-centros en los países de América Latina. Interciencia. 2006;31(4):284-92.

7. Pamo Reyna O. Publicar o Perecer. Bol Soc Per Med Interna. 2000;13(4):180-18.

8. Arroyo-Hernández $\mathrm{CH}$, De la Cruz $\mathrm{W}$, MirandaSoberón UE. Dificultades para el desarrollo de investigaciones en pregrado en una universidad pública de provincia, Perú. Rev Peru Med Exp Salud Publica. 2008;25(4):348-49.

9. Pamo Reyna O, Turín Ramos E, Pacheco Quesada, G. Características de los artículos publicados en la Revista Médica Herediana, 1990-1996. Rev Med Hered. 1998; 9(1):21-27.
10. Pellegrini A. Science for health: notes on the organization of scientific activity for the development of health in Latin America and the Caribbean. Rev Panam Salud Publica.2000; 7(5):345-49.

11. Sanz-Poggi $\mathbf{P}$, Chávez-Solis $\mathbf{P}$, Huamaní $C$, MaytaTristán P. Publicación estudiantil chilena en revistas médicas, 2001-2005. Rev Med Chil [en prensa].

12. Sogi C, Perales A, Anderson A, Zavala S. Calidad de la producción científica de los investigadores de la Facultad de Medicina, UNMSM. An Fac Med (Lima). 2003; 64(2):112-18.

13. Huamaní C, Mayta-Tristán P. Producción científica peruana en medicina y redes de colaboración, análisis del Science Citation Index 2000-2009. Rev Peru Med Exp Salud Publica. 2010; 27(3):315-25.

14. Pamo O. Estado actual de las publicaciones periódicas científicas médicas del Perú. Rev Med Hered. 2005; 16(1):65-73.

15. Ugarte $\mathbf{F}$. La edición de revistas científicas en Latinoamérica. Rev Chil Pediatr. 2004; 75(6):509-11

16. Romaní $\mathbf{F}$, Márquez $\mathbf{J}$, Wong $\mathbf{P}$. Uso de los métodos estadísticos en artículos originales de cinco revistas biomédicas peruanas. Periodo 2002-2009. Rev Peru Epidemiol. 2010;14(2):1-8.

17. Arroyo-Hernández $\mathbf{C H}, \quad$ Angulo-Bazán $\mathbf{Y}$. Publicaciones estudiantiles sobre propiedades medicinales de las plantas en Perú. Rev Peru Med Exp Salud Publica. 2009; 26(4):585-6.

18. Huamaní $\mathbf{C}$, Chávez-Solís $\mathbf{P}$, Mayta-Tristán P. Aporte estudiantil en la publicación de artículos científicos en revistas médicas indizadas en Scielo-Perú, 1997 2005. An Fac Med (Lima). 2008;69(1):42-5.

19. Rojas-Revoredo V. Las publicaciones en revistas indexadas, único indicador de la producción de las sociedades científicas estudiantiles. CIMEL. 2007; 12(1):5-6.

20. Mayta-Tristán P. Sociedades científicas de estudiantes de medicina: el futuro de la investigación en Latinoamérica. MedUNAB. 2010;13(1):3-4.

21. Pérez Jiménez $\mathbf{Y}$, López Flores $M$, Abstengo Jorge Y. Producción científica sobre salud en la provincia de Ciego de Ávila. ACIMED. 1999; 7(2):115-120.

\section{Correspondencia:}

Dr. Hugo Arroyo Hernández.

Correo-electrónico: hugoarroyo12@hotmail.com 